\title{
Jean-Paul HAUTECOEUR
}

sociologue

(1976)

\section{"Nationalisme}

\section{et politique en Acadie"}

Un document produit en version numérique par Jean-Marie Tremblay, bénévole, professeur de sociologie au Cégep de Chicoutimi

Courriel: jean-marie tremblay@uqac.ca

Site web pédagogique : http://www.uqac.ca/jmt-sociologue/

Dans le cadre de la collection: "Les classiques des sciences sociales"

Site web: http://www.uqac.ca/Classiques_des_sciences_sociales/

Une collection développée en collaboration avec la Bibliothèque

Paul-Émile-Boulet de l'Université du Québec à Chicoutimi

Site web: http://bibliotheque.uqac.ca/ 
Cette édition électronique a été réalisée par Jean-Marie Tremblay, bénévole, professeur de sociologie au Cégep de Chicoutimi à partir de :

\section{Jean-Paul HAUTECOEUR}

“Nationalisme et politique en Acadie”. Un article publié dans la revue Recherches sociographiques, vol. 17, no 2, mai-août 1976, pp. 167-188.

L'auteur est sociologue. Il m'a enseigné en 1968 mes cours d'initiation à l'anthropologie à l'Université de Moncton au Nouveau-Brunswick. J'ai aimé. JMT.

Avec l'autorisation formelle de la directrice de la revue Recherches sociographiques, Mme Andrée Fortin, professeure de sociologie à l’Université Laval, qui nous a demandé de bien mentionner nos sources ainsi que la revue qu'elle dirige.

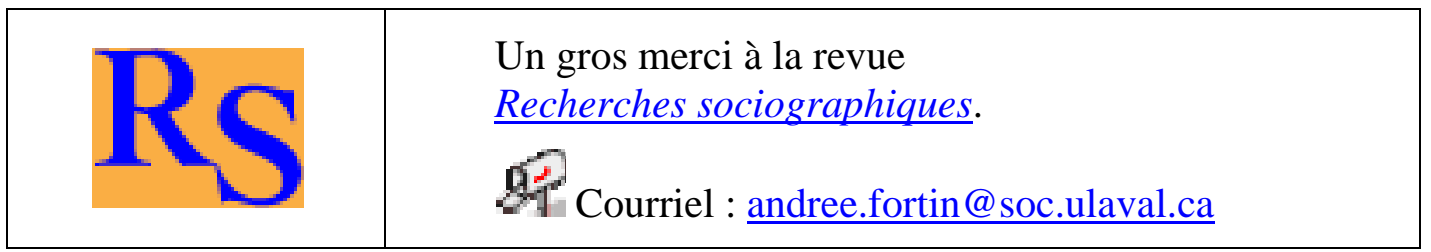

Polices de caractères utilisée :

Pour le texte: Times New Roman, 14 points.

Pour les citations : Times New Roman 12 points.

Pour les notes de bas de page : Times New Roman, 12 points.

Édition électronique réalisée avec le traitement de textes Microsoft Word 2004 pour Macintosh.

Mise en page sur papier format : LETTRE (US letter), 8.5'” $\mathrm{x}$ 11 ')

Édition numérique réalisée le 17 mai 2006 à Chicoutimi, Ville de Saguenay, province de Québec, Canada.

\section{Fait avec}

Macintosh 


\section{Table des matières}

Introduction

I. Recherche d'une objectivité à l'Acadie

A) De quelques indices sociétaux en Acadie

B) Du nationalisme ou du sentiment national

C) De l'idéologie nationale

II. Mythe et histoire

III. Critique du nationalisme acadien 


\section{Jean-Paul HAUTECOEUR (1976) sociologue}

\section{"Nationalisme et politique en Acadie”.}

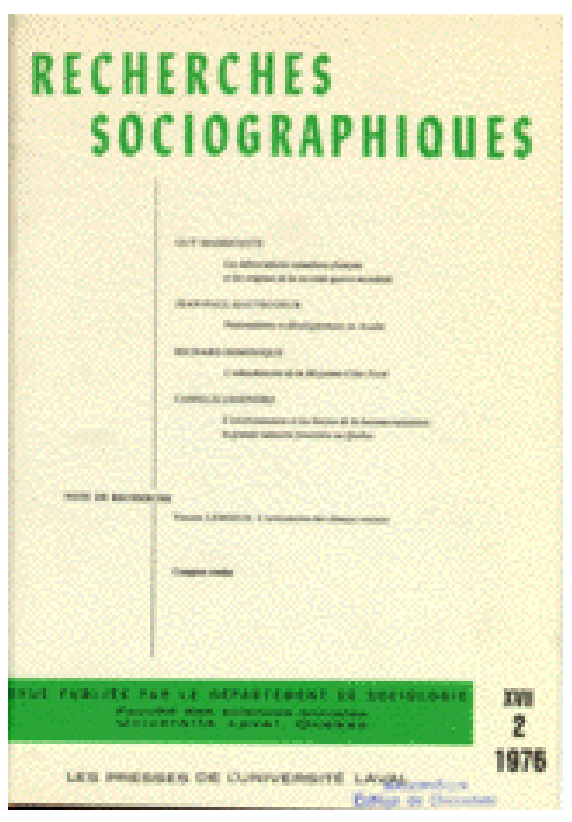

Un article publié dans la revue Recherches sociographiques, vol. 17, no 2, mai-août 1976, pp. 167-188. 
Jean-Paul Hautecoeur,

“ Nationalisme et politique en Acadie” * .

Un article publié dans la revue Recherches sociographiques, vol. 17, no 2, mai-août 1976, pp. 167-188.

\section{Introduction}

$\underline{\text { Retour à la table des matières }}$

Le nationalisme ici considéré est le nationalisme dominant des années soixante que j'ai eu l'occasion d'étudier à partir de sa production idéologique. ${ }^{1}$ C'est le nationalisme officiel explicite de l'élite acadienne en place, généré dans les principales institutions nationales acadiennes et reconnu à l'extérieur de la communauté acadienne comme l'idéologie et le fait constituant de l'actuelle Acadie. Ce nationalisme ne recouvre pas tout le fait nationaliste acadien. Il existe en effet depuis une dizaine d'années un néo-nationalisme (l'expression est des acteurs eux-mêmes) au niveau idéologique comme à celui de la pratique collective. Il est mineur, marginalisé, écarté du prestige

* La présente étude développe certains thèmes d'une conférence donnée à l'Université d'Ottawa dans le cadre d'un colloque sur «les enjeux du développement en Acadie ». Le sujet en était le nationalisme acadien comme facteur de «blocage » ou de " décollage » du développement en Acadie, précisément dans le Nord-est du Nouveau-Brunswick qui est la région à plus forte concentration acadienne. Des interrogations spécifiques sur le sujet général du développement ont été présentées lors du colloque mais ne sont pas reprises dans le cadre de cet article. C'est précisément le nationalisme acadien contemporain qui fait l'objet de l'analyse et sur lequel une précision s'impose.

1 Jean-Paul HAUTECOEUR, L'Acadie du discours, P.U.L., Québec, 1975. 
comme du pouvoir, tenu dans l'illégitimité. Il ne représente pas moins une force réelle et potentielle de régénérescence du nationalisme traditionnel. Dans ce néo-nationalisme, l'imagination est au pouvoir, non plus la tradition. C'est en faisant le pari du pouvoir créateur de ce nationalisme parallèle qui découvre un avenir volontairement objectif à l'Acadie que je me livre à la critique sociologique du nationalisme traditionnel qui prolonge le mythe de l'Acadie et le fait acadien comme survivance. Et c'est en prenant au sérieux le néo-nationalisme encore dans sa genèse qu'il m'est possible de découvrir en Acadie un projet de développement différent de celui du nationalisme dominant. Qu'on n'interprète donc pas le diagnostic fait ici comme une vision morbide de l'histoire. Ce n'est que le diagnostic d'une partie de cette histoire, non d'une fin de partie.

La présente analyse n'est pas seulement un résumé d'études antérieures. Elle y réfère constamment, mais elle est aussi l'occasion d'y ajouter de nouvelles interprétations. Je n'ai pas repris l'analyse amorcée alors du néo-nationalisme. Parce que l'espace d'un article est fait contraignant, surtout parce que depuis 1970 le néo-nationalisme s'est développé dans des manifestations que je n'ai pas eu l'occasion d'observer ou de reconstituer. Cette recherche est à faire avant de pouvoir tirer une conclusion plus globale sur le nationalisme acadien contemporain.

\section{Recherche d'une objectivité à l'Acadie}

$\underline{\text { Retour à la table des matières }}$

Plusieurs approches sont théoriquement possibles quand on cherche à donner de l'Acadie une définition objective, approches qui ont en commun la volonté de dépassement des catégories du sens commun, soit des à priori culturels qui déterminent ce qu'on peut appeler le réalisme naïf des perceptions immédiates. Par analogie avec la constitution ternaire de l'être humain - physique, psychique et cognitif -voici comment on peut caractériser très succinctement ces approches. 
La première consiste, en se situant à l'extérieur, à identifier les indices matériels constitutifs du groupe, de la communauté ou de la société et à leur donner des attributs qui apparaissent spécifiques par comparaison avec d'autres groupes, communautés ou sociétés. Les indices qui viennent immédiatement à l'esprit sont, par exemple, les limites géographiques, la langue, la religion, la production matérielle, le droit, le système politique, la démographie, etc. Cette approche s'attache essentiellement à définir une société comme un objet physique par le nombre, le poids et la mesure. C'est une physiologie sociale. À un niveau élémentaire, c'est aussi l'approche scientifique la moins détachée de ce que j'ai appelé le "réalisme naïf » puisqu'elle consiste surtout à quantifier un objet perçu comme un « donné » à l'observation première.

La deuxième exige de l'observateur qu'il se situe à l'intérieur du groupe ou de la société afin d'y découvrir ses manifestations psychiques, les faits d'âme ou de sentiment, le fait collectif vécu par les acteurs. Ici, c'est l'homme psychique qui devient objet d'investigation, non plus le substrat physique, organisationnel ou institutionnel de sa situation matérielle. Il ne s'agit plus de décrire, mais de comprendre l'être en situation. Comment se manifeste, au niveau sensible, le fait d'être Acadien ? Toutes sortes de constructions sont possibles dans les cadres de cette psychologie sociale ou collective : pour reprendre des démarches classiques, on tentera de définir une "personnalité de base ", un " caractère national ». Mais encore, on pourra pénétrer les faits religieux tels qu'ils sont vécus au niveau du sentiment, et le nationalisme acadien peut être appréhendé à ce niveau.

La troisième approche s'intéresse aux faits de conscience collective, à la production mentale du groupe, de la communauté ou de la société. C'est l'esprit qui est ici objet d'investigation par la médiation du langage, oral ou écrit. Quelle est la définition que les Acadiens donnent de l'Acadie ? On cherchera cette définition comme théorie première dans les idéologies et dans la littérature. Ce nationalisme comme théorie explicite et comme objectivation du sentiment national vécu dans la subjectivité des acteurs peut être appréhendé, au niveau idéologique, comme un fait sociologique déterminant. Mais il ne suffit pas de parcourir pas à pas le discours national en reconstituant métho- 
diquement sa construction et son argumentation pour arriver à sa signification. Le discours est un donné, une théorie première dont il faut analyser forme et contenu, histoire et fonctions. Sa critique doit engendrer une interprétation, pour accéder au fait de connaissance comme théorie seconde.

Les trois types d'approches ainsi caractérisées sont complémentaires, elles peuvent être pratiquées en relative indépendance car leurs méthodes sont différentes. Cependant, le fait social total - dans le cas présent, l'Acadie - ne peut être amputé de l'un de ses niveaux. Une anthropologie générale de l'Acadie devrait s'attacher à décrire la structure de son corps physique, à comprendre les rythmes de son corps animique, à interpréter son Moi manifeste en faits de pensées et de langage. ${ }^{2}$ Le sujet de la présente réflexion sur le nationalisme acadien n'a naturellement pas les dimensions d'une anthropologie générale de l'Acadie. Cependant, pour démontrer la toute importance du nationalisme en Acadie, il est utile d'emprunter chacun de ces chemins, même si le parcours exige une grande économie dans l'exposition.

\section{A) De quelques indices sociétaux en Acadie}

$\underline{\text { Retour à la table des matières }}$

La langue est l'un des indices traditionnels d'identification des Acadiens. S'il reste puissant au niveau idéologique comme thème majeur du nationalisme, il est impossible pratiquement d'en faire une variable statistique objective. Le problème se pose à chaque recensement : si l'on retient la langue maternelle d'origine, on passe à côté de la pratique linguistique réelle. La langue réelle des Acadiens, selon la région, la position socio-professionnelle, le passé scolaire et familial est soit le français, soit l'anglais, soit un usage différencié des deux langues, soit encore un jargon franglais nommé « chiac ». La langue

2 Pour les fondements d'une telle anthropologie, voir: Rudolf STEINER, $\mathrm{Na}$ ture humaine, Paris, Triades, 1970 et Fondements de l'organisme social, Genève, Éd. Anthroposophiques Romandes, 1975. 
française n'est plus un fait collectif d'identification objective de la communauté ou de la société acadienne.

Le même problème se pose quant à l'identification de l'espace sociétal. L'unité significative d'identification spatiale reste la paroisse. La région est déjà un critère ambigu puisqu'il faut retenir des régions a majorité francophone, des régions mixtes et des régions où les Acadiens sont en minorité. S'il est aisé de se représenter le paysage humain habité par une population acadienne, il est néanmoins impossible de représenter l'Acadie géographique comme une unité. L'Acadie n'existe pas sur la carte. ${ }^{3}$

L'Acadie n'a pas de droit. L'Acadie «n'a pas de papiers », comme dirait Morvan Lebesque des Bretons. ${ }^{4}$ Cette langue formelle qui consacre une naissance, une propriété, une histoire, qui confère une existence légale n'existe pas en Acadie. Le droit en vigueur au Nouveau-Brunswick exprime une culture, une histoire et permet de reproduire un ordre social qui appartiennent en propre à la société anglaise. Et qu'il soit traduit ne change rien à l'affaire : l'Acadie est légalement soumise à l'autre, aux autres.

L'Acadie n'a pas d'existence politique. L'institution qui pouvait jadis revendiquer le statut de "parlement officieux des Acadiens », la Société nationale des Acadiens, se définit comme a-politique et par conséquent s'exclut du jeu des partis. Quant au Parti Acadien, on ne peut reconnaître en lui un fait de société globale puisqu'il opère essentiellement dans une région, le Nord-Est du Nouveau-Brunswick, et que jusqu'à date il est demeuré extérieur au parlement de Frédéricton. Le Parti Acadien représente une force politique potentielle d'une histoire à faire ou en-train-de-se-faire, non pas un indice objectif d'identification politique du peuple acadien.

L'Acadie, «capitale du Wellfare » disait Herménégilde Chiasson, est un peuple sous-prolétaire. La production, la distribution, l'organi-

3 « L'Acadie comme lieu, tu ne sais pas où ça commence, où ça finit, qui vit là, quelle langue est parlée » dit Herménégilde CHIASSON interviewé par P. A : Arcand, La Revue, Université de Moncton, mai 1975, p. 136.

4 Morvan LEBESQUE, Comment peut-on être breton ?, Paris, Seuil, 1970. 
sation économiques acadiennes accumulent un ensemble de signes de sous-développement, de dépendance, de domination. ${ }^{5}$ L'infrastructure du mouvement coopératif est insuffisamment développée pour être tenue significative d'une organisation économique originale. Dans ce cas encore, la force idéologique du thème coopératif est plus grande que la force économique réelle. La structure de l'économie acadienne est un indice négatif additionnel. 6

Les écoles ont été de longue tradition un lieu privilégié de transmission de la culture acadienne. L'école et l'église constituaient le cadre institutionnel original de la paroisse acadienne. Or, l'étatisation ou la centralisation de l'éducation (acte des écoles publiques de 1871) ont progressivement modifié le système de transmission du savoir. Le lieu des définitions, des limites et des exclusions du savoir comme de la pédagogie fut transféré au ministère provincial de l'Éducation, avec la conséquence que les enfants acadiens furent progressivement soumis à l'apprentissage des modèles conçus par et pour la société anglaise. L'école comme lieu privilégié de reproduction du système social et d'apprentissage des schèmes idéaux qui le justifient et le fondent est devenue un instrument privilégié d'acculturation, même si dans les régions acadiennes l'enseignement se fait en français. En conséquence et pour notre propos, les écoles ne peuvent figurer comme des institutions acadiennes. ${ }^{7}$

5 Voir : Alain EVEN, Le territoire pilote du Nouveau-Brunswick ou les blocages culturels au développement économique, thèse de doctorat en économie, Université de Rennes, 1970, 4e partie.

6 Le caractère d'extrême généralité de cette exposition est inévitable puisque je ne puis retenir que des grandes conclusions dans le cadre du sujet qui est le nationalisme. Il faut toutefois mentionner que des monographies ou des études plus synthétiques sont extrêmement rares sur l'Acadie, notamment des études économiques contemporaines.

7 Dans ce domaine, des études précises sont aussi nécessaires. Il y a par exemple une École normale de langue française sur le campus de l'Université de Moncton. Dans quelle mesure l'École normale a pour fonction d'initier les maîtres aux mécanismes intégrateurs et aux modèles culturels de la société anglaise ? Dans quelle mesure aussi la participation acadienne au ministère de l'Éducation de Frédéricton contribue à renforcer la domination ou au contraire à freiner l'acculturation? 
Une place à part doit être réservée à l'Université de Moncton et aux institutions spécifiquement "nationales » acadiennes, tout spécialement la Société nationale des Acadiens et des institutions connexes comme le journal L'Évangéline. Ces institutions sont héritières de la longue tradition nationale acadienne et représentent en propre les structures originales de la communauté acadienne. Ce sont les seuls indices objectifs et positifs de production et de reproduction sociétales. L'Église a joué jusqu'à tout récemment un rôle prépondérant et pourrait encore compter parmi les institutions spécifiques du peuple acadien. Pourtant, depuis les années soixante, son rôle et son pouvoir ont été marginalisés dans les limites de la religion, du culte catholique comme cela s'est passé au Québec. Auparavant, l'Église, les institutions nationales et les institutions d'éducation supérieure étaient étroitement confondues. Ce n'est plus le cas. On ne pourrait donc retenir l'Église catholique comme une institution originalement acadienne du fait qu'au Nouveau-Brunswick, Irlandais, Écossais d'origine et autres communautés d'immigrants s'y regroupent également.

En conclusion, l'observateur " extérieur » qui chercherait à identifier l'Acadie comme une société à partir des quelques indices recensés de structure sociétale serait bien forcé de faire un diagnostic radicalement négatif : L'Acadie n'est pas une société. En tant que peuple ou communauté, elle est entièrement intégrée dans les structures de la société anglaise de la province. Ce « corps physique » de l'Acadie, s'il fut un jour objet visible et articulé comme un corps autonome, est aujourd'hui désintégré. Point de densité matérielle ; point de nombre au positif ; point de mesure pour l'arpenteur. Mais c'est peut-être aussi la caractéristique propre de l'Acadie de n'être pas incarnée dans l'espace puisque " c'est la déportation qui est au centre de la réalité acadienne, au centre de l'histoire de l'Acadie ". ${ }^{8}$ Dans ce cas, le physiologue social est destiné à revivre la drôle d'aventure de Don Quichotte en terre de l'absence.

J'ai omis volontairement dans le diagnostic positiviste les institutions nationales ; plus justement, elles ont été laissées entre parenthèses. Or, c'est entre ces parenthèses que s'impose la nation acadienne, entre les fils du canevas de la société anglaise. Les institutions natio-

8 Herménégilde CHIASSON, op. cit., p. 135. 
nales ne constituent pas le squelette d'une société, mais bien la projection matérielle d'un fait sensible qui est dans le corps animique de la nation.

\section{B) Du nationalisme ou du sentiment national}

La nation n'est pas réductible aux signes de société dont il a été traité dans la section précédente. Si l'on a pu assimiler certaines nations à des sociétés achevées - et les contextes historiques particuliers pourraient justifier, en effet, cette réduction ${ }^{9}$ - il est impossible de confondre la nation acadienne et l'Acadie comme société puisque la négation de cette dernière aurait pour corollaire la négation de la nation. Chez les minorités, les sociétés inachevées ou les sociétés en situation coloniale, la nation existe en superposition par rapport aux structures sociales étrangères et à un autre niveau, le psychique ou le pneumatique qui, transposé au niveau des faits de conscience, se manifeste dans la ou les idéologies nationales. Renan situait justement la nation au niveau de l'âme. ${ }^{10}$ Elle est vivante dans le sentiment de la communauté ou du peuple qui adhère à son syncrétisme et c'est en elle que la communauté célèbre les « temps forts » de son rythme collectif.

Être acadien est donc avant tout un fait sensible (différencié d'un fait perceptible par les sens comme d'un fait intelligible) vécu par les

9 Voici par exemple comment Marcel MAUSS définissait une nation dans le contexte européen des années 1920 : « Nous entendons par nation, une société matériellement et moralement intégrée, à pouvoir central stable, permanent, à frontières déterminées, à relative unité morale, mentale et culturelle des habitants qui adhèrent consciemment à l'État et à ses lois [...] Une énorme quantité de sociétés et d'États existent encore dans le monde qui ne méritent à aucun degré le nom de nation [...] Les nations sont les dernières et les plus parfaites des formes de la vie en société. » ( « Nation, nationalité, internationalisme », in Oeuvres, tome III, Paris, Minuit, 1969, pp. 573-639.) [Voir les œuvres de Marcel Mauss dans Les Classiques des sciences sociales. JMT.]

10 Ernest RENAN, «Qu'est-ce qu'une nation ? », in Oeuvres complètes, tome 1, Paris, Calman-Lévy, pp. 887-906. 
acteurs dans l'inspiration. La nation existe à ce niveau de l'air qu'il ne faut pas confondre avec l'idée nationale qui, elle, vit dans l'élément feu ni avec la morphologie sociale (élément terre) dont on découvre la dynamique vivante -biologique - dans l'élément aqueux. ${ }^{11}$ La figure symbolique en qui est représentée la nation acadienne est la Vierge (L'Ave Maris Stella étant l'hymne national acadien) ou encore, dans la légende populaire comme dans le mythe des origines, l'héroïne Évangéline : deux figures féminines, l'une dispensant la grâce comme nourriture céleste reçue dans l'inspiration, l'autre rayonnant l'amour comme rythme harmonique équilibrant entre les dérangements terrestres (voyages) et les perturbations dans les échanges (la déportation) d'une part, les foudres de l'esprit conquérant, organisateur et dominateur d'autre part. Sans lieu à la surface de la terre, sans un système communiquant de canaux qui assurent la cohérence vitale de l'ensemble par une régulière circulation des échanges (fonction liquide), l'Acadie découvre au troisième niveau son lieu unificateur dans le sentiment national qui crée la nation en tant que macrocosme rythmique ou pneumatique intégrant les faits psychiques individuels ou microscopiques.

Il serait de grand intérêt de développer la compréhension de cette Acadianisation dans la direction esquissée, soit dans une psychanalyse des faits d'âme et de ses expressions symboliques appartenant à l'univers aérien. Cette approche aurait l'avantage, se situant dans la dynamique des quatre éléments, de permettre une véritable « compréhension » du nationalisme acadien, compréhension qui échappe aux approches statiques consistant à construire un type abstrait nommé « personnalité de base » ou " caractère national », processus de réification de faits en mouvement. Le plus important ici est d'appréhender la nation comme un fait vécu, donc comme un certain rythme sensible dont il faut trouver les manifestations collectives, les intensités, les fréquences, les nuances intérieures de couleurs, les variations historiques.

Fait intérieur, sentimental ou mystique, le nationalisme est exprime a l'extérieur et collectivement sous la forme d'un culte à l'entité sacra-

11 Voir en particulier les œuvres de Gaston BACHELARD pour une approche de la psychologie par les éléments. 
lisée qu'est la nation. Le sentiment national déborde des expériences du quotidien et du profane en tant que phénomène religieux. Beaucoup d'anthropologues ont traité de cette essence sacrée du nationalisme des minorités, des sociétés déconstruites ou inachevées, en particulier dans les époques de luttes nationales d'indépendance). 12 Même s'il serait abusif de rapprocher les prophétismes islamiques ou les messianismes africains du nationalisme "tempéré » acadien, on doit leur reconnaître de commun leur profonde essence religieuse. En Acadie, le nationalisme et la religion catholique ont toujours été intimement confondus. La nation acadienne est placée «sous la coupole des cieux », la divine Providence veille sur son destin et la mission nationale passe par la dévotion à Marie. «Le religieux compénètre tous les aspects de notre vie nationale », disait Clément Cormier lors d'une assemblée nationale. Un orateur patriote déclarait aussi à l'occasion d'un rassemblement national :

« Mesdames et Messieurs, je ne suis guère porté à faire du sentiment religieux, mais dans une occasion aussi solennelle que celle-ci, je crois qu'il incombe, non seulement à nos évêques et à nos chefs religieux, mais aussi à un laïc de proclamer hautement notre foi profonde en la réalité de l'intervention divine dans notre destin comme peuple et dans la primauté des valeurs spirituelles comme norme de vie nationale [...] N'oublions pas que, par nous-mêmes, nous ne pouvons rien et que nous avons aussi grand besoin du Dieu des nations et de sa sainte Mère qu'au lendemain de la tourmente de $1755 \ldots$.. » 13

En tant que phénomène religieux, le nationalisme acadien comprend un ensemble de signes symboliques, de rites et de dogmes. Parmi les signes symboliques principaux, il faut retenir certains faits historiques mythifiés par la tradition et devenus légendaires, les personnages élevés au rang de figures héroïques et des objets comme le drapeau, des statues religieuses. Les rites nationaux sont rythmés par le calendrier des fêtes annuelles, des fêtes commémoratives et des congrès ou rassemblements patriotiques. Chacune de ces célébrations

12 Voir entre autres, les oeuvres de Georges BALANDIER, A. ABDEL MALEK, Jacques BERQUE, Jean DUVIGNAUD, Franz FANON, pour l'Afrique noire et l'Afrique du Nord.

13 Voir : L'Acadie du discours, op. cit., pour une analyse détaillée du nationalisme sacré. 
est l'occasion de la réactualisation rituelle de la naissance et de la renaissance, des grands périls comme épreuves de mort, aussi du rappel des thèmes mythiques qui doivent être réactualisés oralement pour refaire la continuité du temps historique au plan imaginaire. Enfin, le culte national comprend des dogmes, soit le mythe comme historiographie traditionnelle et l'idéologie nationale dominante dont il est dit explicitement qu'elle doit servir de «bible » à la nation. Quant aux institutions nationales, elles sont des créations sacrées qui ne doivent et ne peuvent par conséquent pas périr : elles remplissent une mission historique de par leur filiation spirituelle et elles devront prolonger cette mission dans l'avenir qui est de «mener l'Acadie jusqu'à ses glorieux sommets ». Il en est de même des hommes dont c'est le destin de servir la cause nationale : porteurs d'une mission sacrée, ils sont entre les dieux et les hommes comme des grands prêtres à qui revient le rôle d'officier au culte national, de redéfinir les dogmes, d'incarner le destin de la nation. Il peut être utile de remarquer l'absence de personnage au charisme prophétique en Acadie. Le nationalisme ne s'est pas construit autour du mythe d'un homme, de son message et de son aura héroïque comme c'est souvent le cas dans les nationalismes messianiques ou de libération. ${ }^{14}$ Il est l'héritage d'une tradition et il revient aux « prêtres » de reproduire cette tradition.

Voilà esquissée cette approche du nationalisme qu'on peut définir comme un fait global d'essence religieuse issu du sentiment national, fait global puisqu'il constitue une collectivité en une nation qu'il faut bien distinguer d'une société même si la nation prétend réaliser l'unitétotalité. C'est en effet la fonction du nationalisme de réaliser l'unitétotalité au plan du sentiment et de l'imaginaire quand c'est la dispersion et le démembrement qui sont les caractères du réel historique. On peut aussi donner au nationalisme une définition plus restrictive en considérant en particulier les idéologies nationales, soit les expressions objectives, explicites du fait national en une doctrine ou une théorie visant à la plus grande cohérence possible et à l'unanimité de

14 Voir : Jean DUVIGNAUD : « Le Destour de Bourguiba a inventé une nation en la nommant et en représentant dramatiquement son malheur puis sa victoire par la personne théâtralisée de son leader. » («Esquisse d'une sociologie du nationalisme tunisien », Les nationalismes maghrébins, Paris, Fondation nationale des sciences politiques, 1966, pp. 83-98.) 
son public. Le nationalisme idéologique cherche à réaliser l'unitétotalité de la nation au niveau du langage et de l'idée de nation. Appréhender le nationalisme à ce niveau de la conscience ou de l'esprit dans la triade définie plus haut, celui du feu dans la dynamique des éléments, est l'objet de la troisième approche.

\section{C) De l'idéologie nationale}

$\underline{\text { Retour à la table des matières }}$

À une époque passée où la «densité morale » de la population acadienne était forte, où le sentiment religieux était intense, où la structure sociale de la paroisse encadrait quasi hermétiquement la vie publique et privée, soit quand la tradition profane et sacrée continuait de régir la vie matérielle comme la vie spirituelle de la communauté acadienne, la fonction du langage articulé en une structure cohérente était essentiellement rituelle, le discours sur la société ou la communauté était donné dans le mythe. ${ }^{15}$ Au risque de simplifier à l'extrême ces époques passées de l'Acadie, on s'en tiendra à cette vision caricaturale pour mieux saisir l'émergence d'une nouvelle forme et d'une nouvelle fonction du langage national à l'époque actuelle.

Si la nation acadienne était jadis un donné, un acquis comme tradition, et si elle était vivante dans le cœur des individus, des familles et des petites communautés, vint un temps où cette forme de solidarité primaire se désagrégea sous la pression d'un ensemble de contraintes, surtout d'origine extérieure, qui eurent pour conséquence des changements d'ordre morphologique, organisationnel, affectif et culturel. L'empreinte des autres sociétés, en particulier de la société anglaise du Nouveau-Brunswick, sur les mœurs et mentalité des Acadiens eurent aussi pour effet de rendre problématique l'identité acadienne et d'affaiblir le sentiment national. La force de syncrétisme de la nation s'est manifestement résorbée. Le sentiment national des leaders patriotes devint un sentiment tragique face aux «capitulations » de la popula-

15 Pour une analyse de l'expression contemporaine de ce mythe, voir : L'Acadie du discours, op. cit., 1er chapitre. 
tion acadienne, à sa " désaffection "pour la cause nationale. ${ }^{16}$ C'est dans cette situation de crise qu'est apparue la nouvelle fonction idéologique du langage national : quand la nation ne s'impose plus comme un donné, il faut bien tenter de la reconstruire si elle est jugée utile au bien collectif. Cette reconstruction est alors tentée au niveau de l'idée ou de l'imaginaire et le discours idéologique aura précisément pour fonction de refaire toute la cohérence et le syncrétisme de la nation dans le discours. On assiste bien dans l'Acadie des années soixante à une "renaissance » du nationalisme, cette fois idéologique par comparaison avec la Renaissance des années 1880 où furent créées les principales institutions nationales.

Dans la recherche d'une objectivité à l'Acadie, la production idéologique des années soixante apparaît à l'observateur comme essentielle. Dans le discours national, l'Acadie s'impose finalement comme une affirmation inconditionnelle et elle accède à la cohérence d'une théorie, soit d'une totalité construite au niveau de l'idée et exprimée par le discours. Pour reprendre une expression chère à Fernand Dumont, on dira qu'à ce niveau de l'esprit, l'Acadie « c'est la théorie que ses membres s'en font ». ${ }^{17}$

Pour conclure, même prématurément puisque dans les deux premières approches exposées précédemment, la recherche est tout proche du point zéro, il faut bien se rendre à l'évidence que le principal, sinon le seul, moyen réel de production des Acadiens est le langage. Le seul lieu d'existence de l'Acadie est l'imaginaire et le sacré : l'imaginaire de la production culturelle et le sacré du nationalisme culturel, la réduction s'effectuant de l'un à l'autre dans le fait total qu'est la na-

16 Id., 2e chapitre, pour de nombreuses expressions acadiennes de ce diagnostic de pathologie de la nation dans les années 55-60.

17 Fernand DUMONT, Les idéologies, Paris, P.U.F., 1974. À côté de la production idéologique et au même titre qu'elle dans la production culturelle figure la production littéraire. Cependant, la production littéraire en Acadie est fait tout récent : elle apparaît dans les années '70. Il serait du plus grand intérêt d'étudier cette nouvelle production du point de vue anthropologique et de la comparer au nationalisme des années '60, de mettre en rapport leurs fonctions identiques et opposées. Car si la littérature est fait idéologique, elle se donne aussi comme un fait anti-idéologique, style individuel irréductible aux productions collectives dominantes. 
tion. Pour revenir aux indices constitutifs d'une société, on comprendra alors que les seules institutions acadiennes originales soient les institutions nationales et les institutions d'éducation supérieure, principalement la Société nationale, le journal L'Évangéline et l'Université de Moncton. ${ }^{18}$ En elles se trouve perpétuellement réexplicitée (retraduite et réinterprétée) la tradition nationale et reproduits les rites de récréation ou de renaissance de la nation.

Il reste à interroger les fonctions réelles du nationalisme acadien dans une perspective de développement. J'ai tenté jusqu'ici de circonscrire l'Acadie actuelle en découvrant la position créatrice du nationalisme dans la constitution de l'Acadie-nation. Comment cette Acadie mythique parvient-elle à prolonger son existence dans l'histoire, à rencontrer l'événement? Un nationalisme de cette nature est-il compatible avec certains projets de développement qui privilégieraient le développement d'une société plutôt que la survivance d'une nation ? Autrement dit, est-ce que le nationalisme acadien traditionnel exerce une action de «blocage » ou de " démarrage » selon une perspective spécifique du développement qu'il faudra également préciser?

18 Voici comment les auteurs du Rapport de la Commission d'enquête sur l'œuvre de la presse acadienne définissaient la mission du journal : « Un journal quotidien français doit apporter aux foyers exposés quotidiennement aux diverses influences anglicisantes la véritable pensée française [...] Dans un tel contexte, il est important qu'une voix se fasse entendre. Cette voix, en quelque sorte la conscience du peuple acadien [...] incitera les élans positifs à la bonne orientation d'un peuple en marche ». (L'Acadie du discours, op. cit., p. 99.) 


\section{Mythe et histoire}

$\underline{\text { Retour à la table des matières }}$

L'Acadie n'est pas une société. Son histoire se résume à une longue dispersion. ${ }^{19}$ Son peuple est divisé en une autre diaspora. Une comparaison avec les sociétés démembrées sous le choc de la Colonisation serait grossière et abusive parce qu'il est problématique de parler d'une société acadienne originaire. Le mythe populaire qu'on retrouve dans l'histographie acadienne a bien créé une société idéale des origines de la colonisation, image du paradis d'avant la chute que suivit le grand cataclysme de la Déportation puis une sorte de Moyen-Âge ténébreux équivalent d'un séjour aux enfers. En fait, l'hypothèse la plus plausible est que la dispersion était à l'origine et que les déportations commandées par l'armée anglaise visaient à empêcher le regroupement des îlots de colonisation, soit la constitution progressive d'une société articulée. L'Acadie, dès l'origine, fut dépossédée du fait historique. Son destin était commandé de l'extérieur et il en est toujours ainsi. Je l'ai montré précédemment : l'Acadie est un organisme sans corps physique intégré et articulé, mal incarné dans les éléments terre et eau, par conséquent réfugié dans la " salle d'attente » de l'âme, univers privilégié du rêve qui sans la force active du corps est aussi séparé de l'univers intelligible de la pensée consciente. C'est donc dans le royaume de l'air, entre le ciel et la terre, que l'Acadie va vivre sa " survivance » et affronter - ou éviter - la dramatique historique.

19 Les déportations géographiques des Acadiens ne sont que des épisodes de cette dispersion. Alors que les déportations étaient commandées de l'extérieur et réfèrent à des événements singuliers, la dispersion est un fait sociologique plus global dont les causes historiques sont à rechercher autant à l'intérieur de la communauté qu'à l'extérieur. Le mythe insiste sur l'événement tragique de la déportation comme retour au chaos primordial ou encore comme renouvellement de la chute. La " dispersion », selon la pensée mythique, devrait cesser avec J'avènement des temps nouveaux : la " Renaissance », passage du chaos au cosmos. De notre point de vue, le phénomène de dispersion caractérise la constance structurelle d'une société au négatif. 
La problématique est alors la suivante : le déplacement, l'exil ou encore le transfert au niveau onirique a-t-il fonction stratégique ou bien se résume-t-il en une conduite d'évitement ? J'emprunterai les mots de Jacques Berque pour poser très justement la question :

« Ainsi la ville se ramassait, dans ce mois de ramadan sous son manteau de fausse inconscience, déchirée par le besoin. Que fallait-il le plus admirer, de son aptitude à ignorer la catastrophe ou de son énergie à la défier ? Telle avait dû être, dans bien des cas, la conduite des sociétés orientales, et de beaucoup d'autres, au moment de l'occupation étrangère. Des peuples rompus se reployaient sur leur prière, qu'on appelait aussi leur illusion. Sur leur désir, sur leur rêve, que j'appelle aussi recours à la Nature et à leur nature.

Leur démarche pouvait aboutir, puisqu'elle les a menés à beaucoup de reprises, malgré la disproportion des forces. Mais dans cette capacité réside un péril : que ce rêve, pour l'appeler ainsi, ne soit pas celui qui donne à l'histoire son envoi, mais l'élude et l'ensevelit. Qu'il ne soit pas la « splendeur orientale »qu'appelait Beaudelaire mais sa caricature d'exotisme et de mensonge, complice de l'agresseur. Et que cette pluralité de recours ne soit pas le jaillissement d'une vérité indivise, mais sa parodie, et non pas une lutte par la métamorphose, mais un lâchage dans la confusion. 20

Je ne reprendrai que brièvement ici un thème qui a déjà été exposé dans des travaux antérieurs. ${ }^{21}$ Étrangère au fait historique, l'Acadie s'est constituée un royaume dans l'univers de la valeur. Il ne suffisait pas que le lieu de son existence soit l'âme ou le rêve, encore fallait-il lui donner à ce niveau une cohérence suffisante pour en faire une totalité, pour réaliser sur ce plan unique l'unité-totalité qui n'est d'ordinaire réalisée qu'en conjonction avec les autres plans ou éléments. Car sans ce travail de totalisation, la désagrégation, même au niveau de l'âme, de l'Acadie eût été accomplie. Donc, face au monopole radical du fait historique des sociétés environnantes ou des systèmes extérieurs, l'Acadie devait s'approprier le monopole radical de sa propre valeur pour opposer à la domination horizontale des autres sa propre maîtrise de la verticale. Alors que les sociétés étrangères pouvaient déterminer

20 Jacques BERQUE, L'Orient second, Paris, Gallimard, 1970, « Préambule ».

21 L'Acadie du discours, op. cit. ; «Les métamorphoses de l'Acadie-nature », La Revue, Université de Moncton. (À paraître.) 
l'événement au moyen de la puissance de production, l'Acadie, elle, pourrait néanmoins définir et orienter le sens de son destin en promulgant l'imaginaire dans le réel, faisant ainsi de l'Acadie une affirmation inconditionnelle et irréductible malgré sa négation dans l'empire du « réel ». Telle est le jeu et l'enjeu du nationalisme acadien. Jeu divin ou simulacre d'un jeu divin transcendant les jeux des hommes car du souffle, du rythme et de la parole devait apparaître le cosmos à la place du chaos où l'unité réelle de la nation s'imposerait comme une victoire sur l'impérialisme de la chose matérielle et du fait historique. Ce « grand œuvre » n'est pas à la mesure des hommes, mais bien des héros, mi-hommes, mi-dieux :

« Nos ancêtres ont été des héros avant, pendant et après la déportation... » 22

Le nationalisme ne peut trouver que dans l'univers sacré cette force prométhéenne. Il est ainsi souvent répété dans les discours nationalistes que la survivance comme la Renaissance ne se seraient pas accomplis sans l'intervention de la divine Providence, que le peuple acadien est sous la protection spéciale de la Mère et de la Vierge Marie, qu'il est porteur d'une mission divine et que ce n'est que par la prière et la dévotion à la patronne des Acadiens que le peuple acadien parviendra à « ses glorieux sommets ».

Pour réaliser cette cohérence suffisante à la création de l'unitétotalité dans l'univers onirique, le nationalisme a besoin du pouvoir absolu du mythe. " Hors du mythe, écrit Denis de Rougemont, il n'y a plus de significations valables dans tous les plans simultanés de notre existence. » Je rappellerai aussi cette analyse de la fonction mythique par Roland Barthes qui est de changer magiquement l'histoire en nature :

« Le mythe a pour charge de fonder une intention historique en nature, une contingence en éternité [...] En passant de l'histoire à la nature, le mythe fait une économie : il abolit la complexité des actes humains, leur donne la simplicité des essences, il supprime toute la dialectique, toute remontée au-delà du visible immédiat, il organise un monde sans contradictions parce que sans profondeur, un monde étalé dans l'évidence, il

22 Anselme CHIASSON, cité dans L'Acadie du discours, op. cit. 
fonde une clarté heureuse : les choses ont l'air de signifier toutes seules. » 23

La renaissance nationale des années soixante est bien une tentative de recréer le mythe de la nation acadienne comme monde sans contradictions, ayant la simplicité des essences, où l'économie de la question permet la grandiloquente affirmation (nationalisme triomphant) et dans lequel le devenir historique revient finalement à la sagesse de la divine Providence. Le mythe se substitue à la science pour donner un sens au monde, il fait l'économie des opérations de l'esprit, ce qui confirme ici l'isolement du nationalisme acadien dans l'élément aérien séparé du feu. Les rituels qui accompagnent le mythe sont aussi des substituts aux actes, à la praxis historique puisqu'il suffit de mimer rituellement la création du monde pour faire ou refaire le monde. Quant aux élus à qui revient le rôle de mystagogues et d'officiers du culte national, ils sont investis naturellement et surnaturellement des pouvoirs de héros. Ce sont les chefs de la nation qui ont fait la Renaissance des années 1880 ; ce sont eux qui ont depuis « mené l'Acadie à ses glorieux sommets ».

Voilà le grand pouvoir magique du mythe national : ériger le fait national - la nation - en une totalité qui, faute d'accès à l'histoire, peut célébrer son épanouissement dans l'imaginaire. Pour le mythe, ce n'est pas la dispersion qui est le signe distinctif de l'Acadie, mais le processus inverse de réunion ou réunification qui est le principe même de la fonction mythique. L'Acadie du nationalisme triomphant des années soixante (né de la commémoration en 1955 du tricentenaire de la Déportation des Acadiens), c'est le mythe de la nation acadienne qu'il avait pour mission de recréer.

Revenons aux belles pages d'introduction à L'Orient second :

«Que de fois n'ont-ils pas dû, contre des rapports écrasants, se faire tous entiers symboles pour résister à la force des choses [...]

« Rêver, c'est mourir peut-être, si cela veut dire lâcher pied devant les duretés de l'action et du combat. Au contraire, si cela veut dire émouvoir

23 Roland BARTHES, Mythologies, Paris, Seuil, 1957, pp. 232-252. 
en soi les possibles, en appeler d'un présent inerte au rapatriement du passé et de l'avenir c'est permettre l'action créatrice. »

L'enjeu du rêve : la mort ou la métamorphose pour ne plus parler de la survivance. La mort, dans l'approche symbolique, est l'issue du nationalisme qui prolonge indéfiniment son existence dans l'univers aérien. Le développement ou la métamorphose, c'est l'acte du nationalisme qui consiste à renouer le fil qui va de la terre au feu, ou encore, par analogie avec l'organisme humain, c'est le rêve en acte qui recherche le poids du corps pour engendrer l'œuvre de l'esprit, son but ultime sans lequel l'Acadie ne pourrait devenir fait réellement intelligible.

La transition du mythique à l'idéologique est opérée dans le nationalisme acadien des années soixante. Tout, dans l'idéologie nationale, n'est pas que rêve et symboles, ce qui serait trop commode pour l'observateur. L'un des acteurs du nationalisme acadien exprimait clairement la dualité :

« Pour éviter de nous laisser entraîner vers l'un ou l'autre extrême, il importe d'appliquer deux règles d'or qui sont les leviers de commande permettant la poursuite du juste milieu. Malgré le paradoxe, je vous énonce d'un souffle ces deux règles : fidélité au passé - rupture avec le passé... Il nous faut donc promouvoir le culte du passé, du moins dans l'ordre de la connaissance et du sentiment. cependant, dans l'ordre de l'action, il existe toujours le danger de s'attacher trop servilement aux formules d'un passé décadent. » 24

Un autre acteur déclarait encore :

« Les formes du nationalisme acadien ont changé avec les générations, c'est vrai. Moi-même, je ne pouvais pas accepter le nationalisme des anciens qui faisaient des discours émus sur la déportation des Acadiens. » 25

Je l'ai dit plus haut: en situation de crise, ici, de la nation, la fonction idéologique est nécessaire, d'une part, pour refaire le syncrétisme des significations, du sentiment et la solidarité des hommes, ce qui

24 Clément CORMIER, cité dans L'Acadie du discours, op. cit., p. 152.

25 Adélard SAVOIE, ibid., p. 117. 
explique le recours à la fonction mythique inhérente à la fonction idéologique, d'autre part, pour définir des projets d'action compatibles avec la nouvelle situation. Le deuxième impératif, s'il implique les fonctions du mythe et des rites, soit la force totalisante de la valeur, suppose néanmoins l'engagement dans le fait historique que Roland Barthes appelle l'intention historique. Et c'est le propre de l'idéologie de chercher à concilier le fait et la valeur alors que le mythe opère la transmutation du fait à la valeur. 26

Le passage est important parce qu'il peut signifier la métamorphose de la nation en une genèse sociétale, ce qui supposerait l'ouverture du nationalisme à la connaissance, à l'imagination créatrice et donc le retour critique à la tradition, aussi son engagement dans des initiatives originales qui auraient pour tâche d'incarner l'Acadie dans son paysage physique (la forme) et de la doter du mouvement propre à l'activité organique. Il s'agit certes d'un passage progressif, historique, et non d'une opération magique qui serait un retour à l'inspiration mythique.

Or, Clément Cormier exprimait clairement la position du nationalisme actuel : promouvoir le culte du passé dans l'ordre de la connaissance et du sentiment, la rupture avec le passé dans l'ordre de l'action. Ce qui signifie : fermer le nationalisme à l'éveil de l'imagination, le confiner dans le domaine subconscient du rêve ; le corollaire : ouvrir le nationalisme au fait historique dont la maîtrise est à l'extérieur, promouvoir la participation acadienne au fait des autres sociétés puisque le fait acadien n'existe pas en dehors de la nation (sentiment national) et qu'il ne peut être créé ou conquis sans la force de l'idée. Ce qui signifie finalement entretien du mythe de la nation pour sa fonction onirique et consécration du statu quo historique dans lequel l'Acadie a statut de "minorité », soit reproduction de la dualité du culturel et du socio-économique, de l'être et du faire.

Le texte même de l'idéologie nationale est explicite sous ce rapport. La « rupture avec le passé » prend forme principalement dans trois directions ou trois projets spécifiques : la consolidation et la multiplication des relations entre l'Acadie et les sociétés environnantes,

26 Voir : Fernand DUMONT, Les idéologies, op. cit., 72-73. 
Canada, Nouveau-Brunswick et Provinces Maritimes; une plus grande participation à la vie économique dans le sens d'une plus grande jouissance dans le Welfare State, une plus grande participation aussi à la vie politique de la province et du Canada sans que les règles traditionnelles du jeu soient remises en cause. ${ }^{27}$ C'est ainsi qu'est tentée la réconciliation du fait et de la valeur dans l'idéologie nationale acadienne : non pas création de l'idée et du fait à partir des multiples possibles que renferme la valeur - la «splendeur orientale » - mais bien conciliation de la valeur avec l'idée et le fait qui viennent d'ailleurs et sûrement assimilation de la valeur interne à la valeur externe, les indices de cette acculturation étant d'ailleurs nombreux dans le discours idéologique.

Le sens de la réponse à la problématique posée est donné. Il reste à articuler l'analyse critique du nationalisme acadien dans le sens d'une contribution possible à un néo-nationalisme qui définirait un projet de développement interne et qui revendiquerait la liberté de définir l'orientation de ce développement. 28

27 Pour une analyse détaillée de ces thèmes, voir : L'Acadie du discours, op. cit., $3 e$ chapitre.

28 Ce néo-nationalisme n'est ni une abstraction, ni un rêve de l'auteur. Il donna dès 1966, naissance à une expression idéologique critique du nationalisme dominant lors du Ralliement de la Jeunesse Acadienne (Voir: L'Acadie du discours, $4 e$ chapitre). Il était en pensée dans le mouvement social qui affecta les principales institutions nationales acadiennes, en 1968-69. Il prit une existence politique dans la création en 1972 du Parti Acadien (Voir : Le Parti Acadien, ouvrage collectif, le Parti Acadien, B.P. 354, Petit Rocher, N.B.) et une nouvelle expression idéologique dans la revue $L$ 'Acayen. Il s'exprime également dans la nouvelle littérature acadienne et dans les mouvements sociaux d'expression populaire, syndicats, coopératives, groupements régionaux. Ce néo-nationalisme devrait faire l'objet d'une étude ultérieure assez systématique. 


\section{Critique du nationalisme acadien}

$\underline{\text { Retour à la table des matières }}$

Le point de vue ou l'approche culturaliste ne peut avoir pour tâche, quand l'intention est de connaissance, de sauver le nationalisme. Privilégier les faits de culture, par décision du sujet comme par "suggestion » de l'objet, est une méthode qui vise avant tout à les comprendre, les réfléchir et tenter de leur donner un sens intelligible. La démarche sociologique est nécessairement critique puisque, prenant les faits de culture comme objet, elle consiste à démonter les constructions premières des acteurs en situation, structurelles comme sémantiques, pour recréer des structures et des significations -constructions secondes - qui ne visent pas les mêmes fins. Les fins de cette critique étant souvent mal identifiées au dehors de l'intention sociologique, le discours sociologique, ici sur la nation et le nationalisme acadiens, est alors réintégré dans le débat idéologique au même titre qu'une idéologie de classe ou de caste. L'intention de la critique n'est donc pas de sauver le nationalisme en adoptant un certain point de vue culturaliste ni de le détruire au moyen de la méthode critique. Se situant dans un projet de connaissance, elle peut espérer tout au plus contribuer au dénouement de la dramatique historique actuelle. ${ }^{29}$

29 Cette sorte de mise au point n'est pas théorique même si elle réfère à certains débats de la communauté des sociologues, par exemple entre écoles marxistes et culturalistes, ou encore entre émules de Marx et émules de Weber. Non. Elle est une forme de dialogue avec des acteurs acadiens imaginaires, aussi un plaidoyer en faveur des intellectuels, de la démarche et de la fonction intellectuelle dans la société acadienne. Ceux-ci sont tout à fait légitimes dans les sciences pures. Mais, la question nationale étant sacrée, domaine réservé aux élites acadiennes, il est quasiment sacrilège que des intellectuels - acadiens et a fortiori non acadiens - s'en emparent pour la poser en d'autres termes, entrant ainsi en concurrence taboue avec l'élite acadienne traditionnelle. L'exil de tels intellectuels de la communauté acadienne, fait indubitable du passé, d'un passé peut-être encore présent, ne peut être prolongé au risque d'un développement unidimensionnel de cette communauté. La question nationale étant 
Sur le plan des valeurs, il faut montrer comment le nationalisme a privilégié certaines valeurs en en excluant d'autres. Dû au caractère profondément religieux du nationalisme, les grandes valeurs qui ont été vénérées appartiennent au domaine moral : le devoir et la dévotion. Devoir d'un peuple martyr porteur d'une mission, devoir aussi tiré du mythe judéo-chrétien de la rédemption ; dévotion à la patronne, à la divine Providence parce que « l'ordre de la grâce vient confirmer et surélever l'ordre de la nature » et que « le principal facteur dans la survivance et le rayonnement du peuple acadien a été et reste encore l'intervention de la toute-puissance divine et la douce protection de notre céleste patronne Notre-Dame de l'Assomption ». 30

J'ai suffisamment insisté sur le fait que le nationalisme se situe surtout au niveau du sentiment (rythme, air) et c'est à celui-ci que la dévotion se manifeste. Quand il passe au niveau de la volonté ou de l'action, la motivation du devoir est fondamentale. Le message vient d'enhaut. Il est ordonnance divine. La hiérarchie des pouvoirs est descendante : les devoirs sont des ordres. On comprend, d'après ce qui précède, que le passage ascendant de l'air au feu soit exclu. L'esprit est inaccessible à l'âme humaine attachée au corps. La dualité est péremptoire tout comme l'impératif moral d'assumer cette dualité. Il n'appartient pas aux hommes de réaliser l'union des contraintes - la coïncidentia oppositorum - pouvoir qui revient aux dieux. ${ }^{31}$

Par conséquent, l'idéal de liberté de l'esprit, idéal grec pré-chrétien réprimé par la suite par la dogmatisation du christianisme, est absent de la structure des valeurs du nationalisme. Cet idéal qui s'applique strictement à l'esprit, soit à l'aventure de la connaissance individuelle et individualisante, est même répressible au nom de l'impératif moral qu'exerce la tradition et le syncrétisme de la nation. On l'a vu, le subs-

au cœur de la destinée de l'Acadie, il est indispensable qu'elle devienne une véritable question et pour cela le rôle des intellectuels est dramatiquement nécessaire.

30 Cité dans L'Acadie du discours, op. cit.

31 Voir en particulier : Mircéa ÉLIADE, Méphistophéles et l'androgyne, Paris, Gallimard, 1962 ; Rudolf STEINER, Goethe et sa conception du monde, Paris, Fischbacher, 1967. Il est impossible, dans l'économie de ce texte, de développer cette analyse même si le risque d'hermétisme est grand. 
titut à l'acte connaissant est le culte au sens donné par la lettre de la tradition. Les discours nationalistes sont la bible de la nation. D'après ce texte et son contexte, l'accès à l'imagination (au sens précis de création d'images comme acte éveillé de la conscience et non pas au sens figuré d'être dans les images à l'état subconscient du rêve) est exclu des normes collectives qui ont comme effet le maintien de la conscience au niveau subconscient, celui du rêve, du sentiment et, au plus subtil, de la mystique nationale.

L'exclusion de l'idéal de liberté au profit d'un idéal de fraternité nationale est lourd de conséquences pour le nationalisme acadien. Le projet de nation, limité aux niveaux du sentiment (dévotion) et de l'action (devoir), occulte le niveau de la connaissance (liberté) d'où pourrait naître un projet de société puisque celle-ci est totalement à imaginer. Cette fonction d'occultation du potentiel spirituel et de rejet de l'élément feu m'apparaît être une force majeure d'anti-développement qui paralyse le nationalisme acadien. Chez la plante, ce niveau occulté serait celui de la fleur et de la graine, la fleur comme manifestation triomphante de son histoire, la graine comme promesse d'accomplir une nouvelle fois le cycle. Si l'on peut dire après Goethe que le but de la feuille est de devenir fleur ${ }^{32}$, on peut dire aussi que le but de la nation est de devenir société. 33

Une autre conséquence importante de l'occultation de l'idéal de liberté dans la vision du monde est la difficulté, sinon l'impossibilité, du passage du nationalisme à l'internationalisme. En d'autres termes, fonder l'identité acadienne dans l'unicité et la subjectivité du sentiment national et ne lui donner pour toute objectivité que l'évidence du mythe revient à condamner l'Acadie à sa solitude et son idéologie à un soliloque. L'ethnocentrisme des peuples ou des communautés à solidarité mythique, surtout quand ceux-ci sont opprimés, est un fait maintes fois rapporté par les ethnologues. Mais ici encore il serait abusif d'étendre l'analogie à l'ensemble des phénomènes. Le syncrétisme du sentiment et du mythe national n'engendre pas le même ethnocentrisme que par exemple la solidarité de type clanique et le totémisme.

32 GOETHE, La métamorphose des plantes, Paris, Triades, 1975.

33 Dans le cas des nationalismes des peuples opprimés, non bien sûr dans celui des nationalismes des sociétés constituées que considérait Mauss. 
C'est parce que le nationalisme acadien est maintenu au niveau subconscient que le sentiment d'identité est vécu comme lieu de l'unique et que la conscience de l'universel lui est inaccessible. Une vision du monde qui a permuté l'idéal de liberté en nécessité - qu'elle soit matérialiste ou providentielle - a aussi exclu l'individuation des possibilités du faire. Or, c'est précisément ce processus d'individuation comme conscience et volonté de réaliser l'unique qui permet en même temps d'embrasser le tout, soit le dépassement de la dualité de l'unique et de l'universel. Le mythe fait la chose par opération magique. La conscience la réalise objectivement par l'union de la chose et de l'idée, la chose nationale n'étant qu'une objectivation particulière de l'idée archétypale. Le nationalisme acadien n'a pu avoir l'intuition de sa solidarité avec d'autres nationalismes comme particularités historiques dans l'évolution générale des civilisations du fait qu'il n'a pu cultiver l'imagination de sa propre individualité historique à cause de son confinement dans l'inspiration, lieu médian du subjectif et du subconscient, royaume du rêve. ${ }^{34}$

On peut reprendre cette analyse d'un point de vue sociologique qui complète l'approche psychanalytique. Il est remarquable que le nationalisme acadien ait exclu de sa rhétorique toute référence au fait de domination. Quand la domination est évoquée, elle est transférée dans l'histoire mythique de la déportation. Or, il suffit d'avoir vécu à Moncton pour savoir que la domination est un fait profondément vécu et d'avoir observé l'Acadie de l'intérieur pour savoir que la domination qui s'exerce sur le fait acadien est profondément inscrite dans les structures sociales et mentales. Rappelons qu'au Nouveau-Brunswick, le nationalisme acadien existe en polarité avec le nationalisme loyaliste et anglophile. Le rapport de domination, pendant le règne du maire Jones à Moncton, était un fait politique explicite et un fait promu au statut de légitimité. ${ }^{35}$ La domination n'est pas, au Nouveau-

34 Pour un exposé détaillé de ces concepts d'imagination, d'inspiration et d'intuition, voir : Rudolf STEINER, Philosophie de la liberté, Paris, Fischbacher, 1963, et Philosophie, cosmologie, religion, Paris, Triades, 1965.

35 Il ne faudrait pas interpréter la politique du maire Jones comme un fait historique singulier, comme une exception attribuable à sa propre personnalité. La domination est un fait historique et structurel touchant toutes les structures sociales. (Voir : Alain EVEN, Les blocages culturels au développement économique, op. cit.) Le légalisme du fait dominant a trouvé son paradigme 
Brunswick, un thème de la rhétorique des intellectuels de gauche, elle est historiquement et structurellement fondamentale.

Il reste à écrire une histoire de l'Acadie sur ces thèmes de la domination, une histoire dégagée de l'apologétique et du mythe nationaliste. Interrogeons plutôt l'absence du thème dans l'idéologie comme fait de structures et fait de classe.

La traditionnelle suprématie du nationalisme en Acadie, des institutions nationales et leur reconnaissance par la société anglaise doivent leur unique possibilité à la règle de « bonne entente » qui normalise la coexistence des deux " sociétés ». Les chefs de la nation acadienne ne sont reconnus comme interlocuteurs valables par ceux de la société anglaise que dans la mesure où ils observent les règles du statu quo. Le nationalisme acadien n'est légitimé à Frédéricton que dans son acception traditionnelle : idéologie de la survivance, rétrécie à la dimension culturelle et surtout linguistique. Que celui-ci manifeste une quelconque velléité politique indépendante du champ politique défini à Frédéricton, il aura des comptes à rendre. ${ }^{36}$ Le « monopole radical du fait historique » exercé en particulier par la société anglaise du Nouveau-Brunswick s'applique naturellement au nationalisme acadien considéré comme fait historique. Le " monopole radical de la valeur » revendiqué par le nationalisme acadien ne peut par conséquent s'exercer que dans l'observation rigoureuse de la différence et dans l'acceptation implicite du monopole horizontal de J'autre. À l'intérieur de ces règles du jeu, il est exclu que le fait de la domination devienne un thème majeur de l'idéologie dominante acadienne. Cette idéologie n'est dominante en Acadie que dans la mesure où elle exclut de son langage le fait de la domination extérieure. Tel est bien l'enjeu : la domination du nationalisme triomphant de l'élite nationaliste acadienne sur la communauté acadienne n'est possible que par l'adoption

contemporain dans la condamnation de Michel Blanchard, emprisonné pour avoir refusé de subir un procès en langue anglaise.

36 Ce qui s'est passé par exemple à l'occasion de la réception par le général De Gaulle des notables acadiens et des accords culturels qui s'ensuivirent. La coopération culturelle fut réintégrée dans les Affaires étrangères du Parlement provincial. 
de l'idéologie et de la norme de «bonne entente » définies par la société dominante.

Il est aisé de déduire cette exclusion de la référence au rapport de domination comme un fait de classe. La petite bourgeoisie nationaliste acadienne, dans sa vision du monde à l'origine de l'idéologie nationale, exclut toute référence aux analyses modernes de la domination. Parce qu'elle est une bourgeoisie elle-même dominante ; parce que, on l'a vu, elle emprunte à l'idéologie extérieure le thème de la «bonne entente »; parce que, on l'a vu également, elle n'a pas de conscience internationale et ne peut donc nouer des solidarités avec d'autres nations opprimées ; parce qu'enfin sa vision morale du monde frappe de l'impératif moral toute idéologie de combat et de libération comme étant incompatible avec le «bien collectif » servi par le devoir et la dévotion. ${ }^{37}$ L'idéal de liberté qui serait à la source d'une analyse de la domination n'est pas un idéal de la bourgeoisie. 38

Il s'ensuit que le statu quo de "minorité » dans une province anglaise est accepté par la petite bourgeoisie acadienne définitrice du nationalisme acadien comme un acquis, comme un fait de nature. Il ne reste qu'à participer plus pleinement au jeu de la «majorité » pour participer également aux bienfaits de l'abondance. D'où le feu vert donné par le nationalisme acadien au développement défini de l'extérieur et pour l'extérieur de l'Acadie. La participation devient la valeur clé dans cette problématique du développement telle que la pose l'élite acadienne.

Fait de structures, fait de classe, l'ouverture du nationalisme acadien à la participation est également favorisé par la conjoncture. Des

37 «Quand on a affaire aux puissants de ce monde, il faut multiplier les prières et les sacrifices auprès de Dieu [...] N'oublions jamais la leçon qui vient de notre passé. La survivance ne serait pas un fait si nos ancêtres s'étaient révoltés contre l'agresseur et avaient eu recours à la violence pour obtenir justice. Leur résignation et leur faiblesse ont attiré sur leur sort les regards de Dieu qui a pris en main leur cause et les a délivrés de leurs ennemis en temps opportun. » (Cité dans L'Acadie du discours, op. cit., p. 134.)

38 Voir en particulier: F. NIETZSCHE, Humain, trop humain, Paris, DenoëlGonthier, 1973, et une très lucide analyse de la vision bourgeoise du monde dans Hermann HESSE, Steppenwolf, London, Pinguin Books, 1959. 
changements ont affecté les institutions nationales et l'élite au pouvoir. L'ancien petit collège Saint-Joseph est devenu l'Université de Moncton, l'ancienne Société l'Assomption une Compagnie mutuelle et elle a donné son nom à une place commerciale de Moncton. Les Acadiens ont suscité un nouvel intérêt à l'extérieur : par l'élection de Louis Robichaud comme premier ministre à Frédéricton, par les travaux de la Commission royale d'enquête sur le bilinguisme et le biculturalisme, par la politique de bilinguisme d'Ottawa, par le voyage quasi diplomatique des notables acadiens à Paris, par le folklore, les œuvres théâtrales d'Antonine Maillet, etc. L'élite nationaliste acadienne a donc vu augmenter son prestige et son pouvoir. Jamais la conjoncture ne fut aussi favorable pour les jeunes diplômés acadiens comme pour la petite bourgeoisie professionnelle à qui des postes sont ouverts dans l'administration des secteurs public et privé. La participation technologique est encouragée tant dans les Provinces Maritimes qu'à Ottawa dans les organismes fédéraux. Dans les lignes de l'idéologie nationale on peut lire : " nous vivons une période de notre histoire qui en est une de révolution... Il semble qu'un vent de saine révolution ait soufflé sur la nation. » 39

Encouragée de l'extérieur, la participation acadienne aux faits économique, politique et technologique des sociétés environnantes est aussi encouragée par le nationalisme acadien. ${ }^{40}$ L'intérêt de classe n'est même pas a souligner. Il est identifié à celui de la nation. Ainsi, saisissant une conjoncture favorable, la petite bourgeoisie nationaliste se faisait le porte-parole de projets sociétaux définis à l'extérieur de la communauté acadienne, consacrant la participation acadienne à la domination extérieure qui nie l'Acadie comme société, sinon réelle, du moins possible.

Le dernier thème de l'analyse découle des précédents et en sera la conclusion : le nationalisme acadien, en faisant un projet collectif en termes de nation, devait exclure comme incompatible à ses fins tout

39 Cité dans L'Acadie du discours, op. cit., p. 126.

40 Il est intéressant de noter ici que l'Université de Moncton, dont la direction était aux mains de la petite bourgeoisie nationale, décernait en 1970 un doctorat honorifique à M. K. C. Irving, homme d'affaires florissantes originaire du Nouveau-Brunswick et symbole triomphant de l'entrepreneur capitaliste. 
projet de société qui aurait été défini à l'intérieur de la communauté acadienne et pour elle-même. La structure de domination est implacable. La tradition du nationalisme acadien est lourde. Mais aussi, les intérêts de classe de la bourgeoisie montante animés par une conjoncture favorable à ses fins ont fait naître un nouveau pouvoir, le pouvoir de la petite bourgeoisie montante, pouvoir extra-nationaliste mais aussi héritier du pouvoir nationaliste traditionnel et toujours confondu avec lui. Les leaders de l'ancien nationalisme étaient - je le suppose car ce type d'analyse historique n'a jamais été fait à ma connaissance l'élite patriotique recrutée surtout dans le clergé, chez les enseignants et dans les «professions ». Les définiteurs du nationalisme des années soixante, ces mêmes patriotes à l'origine, ont hérité comme tels de la tradition mais ont aussi assisté aux changements mentionnés. Ils les ont assumés à la tête des principales institutions acadiennes comme au sein des institutions non acadiennes auxquelles ils ont été amenés à participer. Ces changements ont affecté l'élite elle-même en favorisant l'émergence d'une nouvelle classe en Acadie, la petite bourgeoisie. Cette nouvelle classe a beaucoup en commun avec les petites bourgeoisies des autres sociétés avec qui elle est venue en contact. Néanmoins, elle s'en distingue par le fait spécifique d'être l'héritière de l'ancienne élite nationaliste, de son idéologie comme des institutions nationales.

C'est dans ce fait qu'il faut rechercher l'ambiguïté ou la contradiction de l'actuel nationalisme. D'une part, la petite bourgeoisie adhère aux valeurs de cette classe, quelle que soit l'origine culturelle, elle fait sienne l'idéologie technologique des sociétés post-industrielles et adopte de nombreux modèles culturels de ces sociétés. D'autre part, elle est identifiée et s'identifie toujours à l'ancienne élite nationaliste porteuse de la mission nationale, définitrice du mythe de la nation et unanime représentant de l'intérêt collectif. L'un de ses rôles va être de résoudre la contradiction et c'est là sa fonction idéologique : concilier, rendre compatibles le projet de classe et le projet national. Le projet intérieur de nation doit nécessairement s'accommoder du projet extérieur de société trans-nationale. Mais plus encore, le projet de nation devrait servir l'intérêt de classe et le projet de société post-industrielle trans-nationale, comme on voudra bien l'appeler, servir à rénover l'ancien mythe de la nation qui ne peut plus s'appuyer pour exercer toutes ses fonctions sur l'ancienne unanimité de la communauté. Et c'est ainsi 
que le nationalisme acadien est amené à définir la nation acadienne indépendamment d'une volonté d'incarner cette nation dans des structures sociales originales.

C'est donc un fait relativement nouveau que cette appropriation en Acadie du nationalisme par une classe dont les intérêts ne s'identifient pas à ceux de la communauté acadienne comme société potentielle et possible.

« Le nationalisme est valable tant qu'un peuple est opprimé : alors il rassemble dans une aspiration toutes les forces sociales diverses, également humiliées et espérantes ; mais lorsque ce peuple est libéré, en mesure de débattre de ses propres affaires, le nationalisme ne peut plus fournir de réponse sérieuse aux problèmes réels, rien que des excitations stériles et des contradictions indéfinies. Il devient l'alibi des privilégiés qui recourent au mythe de la « totalité nationale » pour faire oublier les inégalités réelles. Du nationalisme des peuples indépendants, il est injuste de tirer argument contre le nationalisme des peuples opprimés. On n'en aperçoit pas moins le danger qu'un nationalisme né dans l'oppression ne devienne ensuite le moyen démagogique d'éluder les tâches de la construction économique et de l'égalité sociale... » 41

Le nationalisme acadien a laissé tomber une étape, et la plus importante : celle de libération de l'énergie refoulée en un acte de création d'une société possible. En occultant sa fonction, il est effectivement devenu un nationalisme de l'oppression, complice, non pas de l'agresseur car le contexte n'est pas identique au contexte colonial, mais du fait de la domination des sociétés et des cultures fortes.

Les faits de répression exercés par la petite bourgeoisie nationaliste acadienne sur les expressions émergentes du néo-nationalisme porteur d'un projet de société ont été particulièrement manifestes dans les années passées. On citera le sort qui fut réservé aux intellectuels acadiens qui organisèrent le Ralliement de la jeunesse francophone en 1966 ; le même sort qui fut réservé aux intellectuels et leaders étudiants du mouvement néo-nationaliste qui affecta l'Université de Moncton et les principales institutions nationales en 1968-69 ${ }^{42}$; la

41 Jean-Marie DOMENACH, «Les nationalismes et le dépassement des nations », Esprit, mars 1955.

42 Voir : L'Acadie du discours, op. cit., 4e et 5e chapitres. 
politique d'isolement et de marginalisation d'institutions parallèles telles que le Parti Acadien, des fédérations régionales comme la Fédération des Acadiens du Nord-Est, des centres régionaux d'animation sociale comme le CRAN ; la consigne du silence et la politique de tampon à l'occasion de manifestations contre le pouvoir politique, linguistique ou juridique de Frédéricton ; la politique anti-syndicale de la petite bourgeoisie à l'occasion de formation d'unités syndicales dans les institutions nationales ou extra-nationalistes 43 et comme moyen de totalisation du pouvoir à tous les niveaux de la vie nationale, le contrôle systématique des lieux de décisions et l'intégration des nouvelles élites dans les rangs de la " patente ». ${ }^{44}$ Le pouvoir totalisant du mythe de la nation comme mécanisme de réduction de la diversité à la totalité se fait pouvoir totalitaire quand l'unité-totalité créée dans l'imaginaire ou dans le discours est niée par les différences, les conflits et les contradictions de la praxis collective.

En Acadie, le nationalisme dominant a ouvert une voie au développement défini à l'extérieur par les classes dominantes en fermant les voies de développement diversement définies de l'intérieur ou demeurées indéfinies parmi un peuple dispersé, dépossédé de ses moyens de production. Le nationalisme dominant a monopolisé, non seulement la valeur, mais également le fait potentiel pour réaliser les aspirations d'une nouvelle classe, la petite bourgeoisie. Ces aspirations sont à l'image de celles des petites bourgeoisies des sociétés " développées ». L'esprit y est expulsé comme il l'est du mythe de la nation acadienne. Et pour toutes ces raisons, la visée-vision d'une société acadienne a été refoulée. En Acadie, le projet de nation a baillonné le projet de société. Si l'histoire devait suivre ce cours, l'Acadie serait irrémédiablement condamnée au spectacle, ce qu'elle est déjà partiel-

43 Voir : La revue L'Acayen où certains de ces faits sont relatés et analysés.

44 La «Patente » est le nom donné par les Acadiens à l'Ordre Jacques-Cartier ou à ce qu'il en reste au Nouveau-Brunswick. Pouvoir à connotation occulte et magique au niveau populaire, attribut d'une mythique société secrète, la Patente n'existe pas moins réellement en tant que structure de pouvoir totalisant et totalitaire, démocratique en façade mais essentiellement hiérarchique, s'exerçant par la contrainte indirecte et usant toujours de l'intérêt national comme moyen de légitimation, La "Patente » est également personnalisée dans les quelques figures bien connues qui, dans les années '60, formaient l'élite nationaliste. 
lement devenue : chose pittoresque dans le canevas de l'industrie touristique, prétexte au rêve ou à l'orgie.

\title{
Jean-Paul HAUTECOEUR
}

\author{
Fin du texte
}

